\title{
The Effect of Renal Impairment on the Pharmacokinetics and Safety of Talazoparib in Patients with Advanced Solid Tumors
}

\author{
Chandrasekar Durairaj ${ }^{1}$. Jayeta Chakrabarti ${ }^{2} \cdot$ Cristiano Ferrario $^{3} \cdot$ Holger W. Hirte $^{4} \cdot$ Sunil Babu $^{5}$. \\ Sarina A. Piha-Paul ${ }^{6}$. Anna Plotka ${ }^{7}$. Justin Hoffman ${ }^{1} \cdot$ Haihong Shi $^{8} \cdot$ Diane D. Wang ${ }^{1}$
}

Accepted: 27 December 2020 / Published online: 9 March 2021

(c) The Author(s) 2021

\begin{abstract}
Background Pharmacokinetic (PK) studies suggest that talazoparib is primarily eliminated unchanged via renal excretion. The current study investigated how varying degrees of renal impairment may affect the PK of talazoparib, and evaluated the safety and tolerability of talazoparib, in patients with advanced solid tumors with/without renal impairment.

Methods Patients with advanced solid tumors and normal renal function or different degrees of renal impairment measured by estimated glomerular filtration rate (eGFR: mild $=60-89$, moderate $=30-59$, severe $=15-29 \mathrm{~mL} / \mathrm{min} / 1.73 \mathrm{~m}^{2}$ ) were enrolled in this open-label, non-randomized, phase I study. Talazoparib was administered orally at $0.5 \mathrm{mg} /$ day for 22 days. Primary PK parameters included the area under the plasma concentration-time curve from 0 to $24 \mathrm{~h}\left(\mathrm{AUC}_{0-24}\right)$ and maximum observed plasma concentration $\left(C_{\max }\right)$ at steady state (Day 22). Safety and tolerability were also investigated.

Results Thirty-four patients were enrolled. At Day 22, compared with patients with normal renal function $(n=9)$, patients with mild $(n=9)$, moderate $(n=8)$, or severe $(n=8)$ renal impairment had a $12.2 \%, 43.0 \%$, and $163.3 \%$ increase in talazoparib $\mathrm{AUC}_{0-24}$, and a $11.1 \%, 31.6 \%$, and $89.3 \%$ increase in talazoparib $C_{\max }$, respectively. Talazoparib was generally well tolerated, and overall there were no notable differences in the treatment-emergent adverse event profile across renal function groups.

Conclusions Exposure to talazoparib increased with worsening renal impairment. Overall, this study confirms current dosing recommendations in patients with mild and moderate renal impairment $(1 \mathrm{mg} / \mathrm{day}$ and $0.75 \mathrm{mg} / \mathrm{day}$, respectively) and indicates that a lower starting dose of $0.5 \mathrm{mg} /$ day should be considered for patients with severe renal impairment.
\end{abstract}

Clinical Trials Registration NCT02997163.

Diane D. Wang

Diane.Wang@Pfizer.com

1 Clinical Pharmacology, Pfizer Inc., 10555 Science Center Drive, San Diego, CA 92121, USA

2 Pfizer Ltd, Surrey, UK

3 Jewish General Hospital, McGill University, Montreal, QC, Canada

4 Juravinski Cancer Centre, Hamilton, ON, Canada

5 Fort Wayne Medical Oncology and Hematology, Fort Wayne, IN, USA

6 Investigational Cancer Therapeutics, The University of Texas MD Anderson Cancer Center, Houston, TX, USA

7 Pfizer Inc., Collegeville, PA, USA

8 Pfizer Inc., Groton, CT, USA

\section{Introduction}

Talazoparib is an inhibitor of poly(ADP-ribose) polymerase (PARP) enzymes 1 and 2 [1]. By inhibiting the catalytic activity of PARP1 and PARP2, and by trapping PARP on single-strand DNA breaks, talazoparib prevents DNA repair, thereby selectively killing tumor cells with DNA damage repair (DDR) pathway defects, including those with germline breast cancer susceptibility genes (BRCA) 1/2 alterations [1-3]. Talazoparib $1 \mathrm{mg}$ once daily has been approved in multiple countries as monotherapy for the treatment of patients with deleterious or suspected deleterious germline BRCA1/2-mutated, human epidermal growth factor receptor 2-negative advanced breast cancer $[4,5]$. This approval was based on the results of a pivotal phase III trial (EMBRACA), where patients treated with talazoparib had a statistically significant and clinically meaningful improvement in progression-free survival and patient-reported outcomes compared with physician's choice of chemotherapy [6, 7]. Treatment with talazoparib was well tolerated, with anemia, 


\section{Key Points}

Studies assessing the pharmacokinetics (PK) of talazoparib suggest that renal excretion of unchanged drug is the major route of talazoparib elimination. This Phase I study assessed the impact of renal impairment on the PK of talazoparib in adult patients with advanced solid tumors and varying degrees of renal dysfunction.

Following administration of multiple $0.5 \mathrm{mg}$ doses of talazoparib, the steady-state exposure of talazoparib increased with worsening renal impairment. Talazoparib exposure increased by $12.2 \%, 43.0 \%$, and $163.3 \%$ in patients with mild, moderate, or severe renal impairment, respectively, when compared with patients with normal renal function.

Overall, this study confirms current dosing recommendations in patients with mild and moderate renal impairment ( $1 \mathrm{mg} /$ day and $0.75 \mathrm{mg} /$ day, respectively) and indicates that a lower starting dose of $0.5 \mathrm{mg} /$ day should be considered for patients with severe renal impairment. Talazoparib was generally well tolerated, and overall there were no notable differences in the treatment-emergent adverse event profile across renal function groups.

fatigue, and nausea being the most common adverse events (AEs) $[7,8]$. Grade 3-4 AEs were primarily hematologic and occurred in 55\% of patients taking talazoparib [7]; however, AEs could be managed by dose modifications and only $1.4 \%$ of patients permanently discontinued talazoparib treatment due to hematologic toxicities [7, 8].

The pharmacokinetic (PK) profile of talazoparib has been characterized in prior clinical studies conducted in patients with advanced tumors [9-12]. In these studies, talazoparib was rapidly absorbed following oral administration, with a median time to first occurrence of maximum observed plasma concentration $\left(T_{\max }\right)$ ranging from approximately 1.0 to $2.0 \mathrm{~h}$ postdose $[9,11]$. Talazoparib exhibited linear PK across the dose range of $0.025-1.1 \mathrm{mg}$ once daily [11], and steady state was reached after 2-3 weeks of daily dosing $[11,13]$. Results from a mass balance and metabolic profiling study in patients with advanced solid tumors indicate that talazoparib undergoes minimal hepatic metabolism $[10,13]$. Following administration of a single oral dose of ${ }^{14} \mathrm{C}$-talazoparib, a mean of $68.7 \%$ and $19.7 \%$ of the total administered radioactive dose was recovered in urine and feces, respectively [10]. Excretion of unchanged talazoparib in urine was the major route of elimination, accounting for
$54.6 \%$ of the administered dose, while unchanged talazoparib recovered in the feces accounted for $13.6 \%$, indicating that renal excretion plays a major role in the elimination of unchanged talazoparib [10]. Impaired renal function may alter the PK of drugs primarily eliminated by renal excretion to an extent that the recommended dose may differ from the dose used in patients with normal renal function.

Consistent with these findings, a population PK analysis using pooled data from multiple clinical studies of talazoparib that included 490 patients, where 132 patients had mild renal impairment $\left(60 \mathrm{~mL} / \mathrm{min} \leq\right.$ creatinine clearance $\left[\mathrm{CL}_{\mathrm{cr}}\right]$ $<90 \mathrm{~mL} / \mathrm{min}$ ), 33 patients had moderate renal impairment ( $30 \mathrm{~mL} / \mathrm{min} \leq \mathrm{CL}_{\mathrm{cr}}<60 \mathrm{~mL} / \mathrm{min}$ ), and 1 patient had severe renal impairment $\left(\mathrm{CL}_{\mathrm{cr}}<30 \mathrm{~mL} / \mathrm{min}\right)$, showed that talazoparib apparent oral clearance (CL/F) was decreased by $15 \%$ and $38 \%$ in patients with mild and moderate renal impairment, corresponding to an $18 \%$ and $61 \%$ increase in total exposure, respectively, when compared to patients with normal renal function $\left(\mathrm{CL}_{\mathrm{cr}} \geq 90 \mathrm{~mL} / \mathrm{min}\right)$ [13]. In addition, a pooled safety analysis was conducted in 494 patients treated with talazoparib $1 \mathrm{mg}$ once daily, where patients were also grouped based on $\mathrm{CL}_{\mathrm{cr}}$ [14]. Among these patients, $60.7 \%$ had normal renal function at baseline, $31.8 \%$ had mild renal impairment, $7.3 \%$ had moderate renal impairment, and $0.2 \%$ (one patient) had severe renal impairment. While the AE frequencies were generally comparable between patients with mild renal impairment and those with normal renal function, $\mathrm{AE}$ frequencies were higher among patients with moderate renal impairment [14]. Based on these findings, the current dosing recommendation is to reduce the dose of talazoparib to $0.75 \mathrm{mg}$ once daily in patients with moderate renal impairment $[4,5]$; however, due to the insufficient number of patients $(n=1)$, the impact of severe renal dysfunction could not be estimated [14]. These results clearly indicate that patients with impaired renal function are at risk of higher talazoparib exposure, consistent with renal excretion of unchanged talazoparib being the major route of elimination for talazoparib. Therefore, it is important to evaluate the potential effect of renal dysfunction on the PK and safety of talazoparib as it is likely to be administered to patients with various degrees of renal impairment.

The current study was a dedicated study to further evaluate the effect of varying degrees of renal impairment on the PK of talazoparib, and to assess the safety and tolerability of talazoparib, in patients with advanced solid tumors with or without renal impairment. 


\section{Materials and Methods}

\subsection{Study Design and Treatment}

This was an open-label, non-randomized, multicenter, phase I study (NCT02997163) designed to compare the PK and safety of talazoparib in patients with advanced solid tumors and impaired renal function to those with normal renal function. Eligible patients were enrolled in parallel and were assigned to one of four groups based on investigator sitereported renal function at the enrollment visit on Day 1, as measured by estimated glomerular filtration rate (eGFR), calculated using the Modification of Diet in Renal Disease (MDRD) formula: normal renal function (eGFR $\geq 90 \mathrm{~mL} /$ $\mathrm{min} / 1.73 \mathrm{~m}^{2}$ ), mild renal impairment (eGFR $\geq 60$ and $\leq 89$ $\mathrm{mL} / \mathrm{min} / 1.73 \mathrm{~m}^{2}$ ), moderate renal impairment (eGFR $\geq 30$ and $\leq 59 \mathrm{~mL} / \mathrm{min} / 1.73 \mathrm{~m}^{2}$ ), and severe renal impairment (eGFR $\geq 15$ and $\leq 29 \mathrm{~mL} / \mathrm{min} / 1.73 \mathrm{~m}^{2}$ ). Patients received 22 days of treatment with talazoparib $0.5 \mathrm{mg}$ once daily (administered orally as two $0.25 \mathrm{mg}$ capsules), which is $50 \%$ of the recommended dose for patients with $\mathrm{CL}_{\mathrm{cr}} \geq 60 \mathrm{~mL} /$ $\mathrm{min}$. The reduced dose of $0.5 \mathrm{mg}$ provided a twofold safety margin relative to the maximum tolerated dose $(1 \mathrm{mg})$ to account for the potential increase in talazoparib exposure in patients with renal impairment. Patient enrollment was continued until there were at least six PK-evaluable patients (see Sect. 2.4) in each of the four study groups. Talazoparib dose modifications due to AEs (either grade 3 or 4 hematologic toxicities or other grade 3 or 4 events as agreed between the investigator and medical monitor) and liver test abnormalities (as specified in US FDA Guidance for Industry, DrugInduced Liver Injury) were permitted in the study.

\subsection{Patient Eligibility}

Eligible patients were aged $\geq 18$ years and had histological or cytological confirmed advanced solid tumors with no standard approved treatment options, an Eastern Cooperative Oncology Group (ECOG) performance status $\leq 2$, and a life expectancy $\geq 3$ months. Patients were required to have no clinically significant change in renal status within 3 months of enrollment and renal function must have remained stable between screening and enrollment $(\leq 25 \%$ change in eGFR for patients with mild or moderate renal impairment or $\leq 30 \%$ for patients with severe renal impairment). Renal function classification was based on the assessment made at enrollment if the screening classification differed. If patients were taking concurrent medication for the treatment of renal impairment, the dose had to have been stable for $\geq 1$ month prior to enrollment and was expected to remain stable throughout the study.
The main exclusion criteria included treatment with a systemic anticancer therapy within 14 days or five halflives (whichever was longer) prior to enrollment, or use of a P-glycoprotein inhibitor or inducer, or an inhibitor of breast cancer resistance protein, within 7 days or five half-lives (whichever was longer) prior to day 1. Patients currently receiving hemodialysis and/or peritoneal dialysis and those with gastrointestinal disorders likely to affect drug absorption were also excluded.

\subsection{Outcomes and Assessments}

\subsubsection{Pharmacokinetic Assessments}

Serial blood samples for determination of talazoparib in plasma were collected predose, and at $0.5,1,2,4,6,8-12$, and $24 \mathrm{~h}$ postdose on Day 1 (single dose) and Day 22 (multiple dose). Additional blood samples were collected at predose on Days 8 and 15. Urine samples for PK analysis were collected as a single void at predose on Day 1 , and all urine voided after talazoparib dosing on Day 1 and Day 22 was collected for voids between intervals of $0-12 \mathrm{~h}$ and $12-24 \mathrm{~h}$. In addition, separate blood samples were collected at $2 \mathrm{~h}$ postdose on Days 1 and 22 for plasma protein binding evaluation. Plasma and urine talazoparib PK parameters were calculated using the standard non-compartmental analyses with Phoenix WinNonlin (version 8.1; Certara USA, Princeton, NJ, USA). Primary PK parameters were the talazoparib area under the plasma concentration-time curve from 0 to $24 \mathrm{~h}$ $\left(\mathrm{AUC}_{0-24}\right)$, maximum observed plasma concentration $\left(C_{\text {max }}\right)$, unbound $\mathrm{AUC}_{0-24}\left(\mathrm{AUC}_{0-24 \mathrm{u}}\right)$, and unbound $C_{\max }\left(C_{\operatorname{maxu}}\right)$ at steady state (Day 22). Secondary PK parameters were the talazoparib plasma $T_{\max }$, predose concentration $\left(C_{\text {trough }}\right)$, $\mathrm{CL} / \mathrm{F}$, accumulation ratio $\left(R_{\mathrm{ac}}\right)$, fraction of unbound drug in plasma $\left(f_{\mathrm{u}}\right)$, unbound $\mathrm{CL} / \mathrm{F}\left(\mathrm{CL}_{\mathrm{u}} / \mathrm{F}\right)$ at Day 22 , amount of drug excreted in urine from 0 to $24 \mathrm{~h}$ postdose $\left(\mathrm{Ae}_{0-24}\right)$, percentage of dose excreted in urine $\left(\mathrm{Ae}_{0-24} \%\right)$, and renal clearance $\left(\mathrm{CL}_{\mathrm{r}}\right)$ at Day 22. Secondary PK parameters also included single-dose values in plasma $\left(\mathrm{AUC}_{0-24}, C_{\max }, T_{\max }\right.$, $f_{\mathrm{u}}, \mathrm{AUC}_{0-24 \mathrm{u}}$, and $\left.C_{\text {maxu }}\right)$ and urine $\left(\mathrm{Ae}_{0-24}\right.$ and $\left.\mathrm{Ae}_{0-24} \%\right)$.

Validated high-performance liquid chromatography tandem mass spectrometric (HPLC-MS/MS) methods were used to determine the concentration of talazoparib in plasma and urine, and to determine the extent of protein binding of talazoparib in plasma and dialysate fluid (Alliance Pharma Inc., Malvern, PA, USA). See the electronic supplementary materials for a description of bioanalytical methods and assay performance.

\subsubsection{Safety Assessments}

The safety of talazoparib was evaluated based on the incidence of AEs, including serious AEs (SAEs), the severity 
of AEs, the incidence of dose modification and permanent discontinuation due to AEs, and the incidence of clinically significant changes in vital signs, electrocardiograms (ECGs), and clinical laboratory values. Treatment-emergent safety data were collected from the first dose of talazoparib through 30 days after the last dose, before initiation of a new antineoplastic therapy or enrollment into an open-label extension (OLE) study (NCT02921919), whichever occurred first. A treatment-emergent AE (TEAE) was considered to be treatment related if it had at least a possible relationship to talazoparib, as assessed by the investigator, or was missing a causal relationship assessment and for which a relationship to the study drug could not be excluded. The Medical Dictionary for Regulatory Activities version 21.1 was used to code AEs and the severity of TEAEs was graded according to the Common Terminology Criteria for Adverse Events (CTCAE) version 4.03. In addition, renal function assessments, including body surface area (BSA)-normalized eGFR, absolute eGFR, and $\mathrm{CL}_{\mathrm{cr}}$ were retrospectively estimated using the laboratory values collected on Days 8, 15, and 22 as part of safety assessments.

A safety follow-up occurred 30 days after the last dose of talazoparib or before starting a new antineoplastic therapy, whichever happened first. Assessments included vital signs, physical examination, 12-lead ECG, ECOG performance status, AE and medication review, serum chemistry, and hematology. For eligible patients who continued talazoparib in the OLE study, collection of TEAEs for this report continued only until the 30-day follow-up safety visit.

\subsection{Statistical Analysis}

A sample size of approximately 24 patients was planned to ensure that at least six patients with evaluable PK data in each renal function group completed the study. The sample size was based on the recommendation from the European Medicines Agency's guideline on the evaluation of medicinal products in patients with decreased renal function [15].

The PK population was defined as all patients who had at least one reportable talazoparib concentration. The PK analysis population was defined as all PK evaluable patients who provided at least one of the PK parameters of primary interest; patients in this population were considered PK evaluable if they met the following criteria: completed 22 calendar days of treatment with talazoparib (regardless of any treatment hold) and missed $\leq 5$ consecutive doses; received $\geq 10$ consecutive days of talazoparib $0.5 \mathrm{mg}$ daily dose without dosing interruption prior to Day 22; completed $\geq 85 \%$ of total plasma PK sample collections; and had not vomited talazoparib dose on Days 1 and 22 of the PK sample collection. PK parameters were summarized descriptively by renal function group for the PK analysis population. Descriptive statistics were calculated for PK parameters available from three or more patients.

The PK parameters $\mathrm{AUC}_{0-24}, C_{\max }, \mathrm{AUC}_{0-24 \mathrm{u}}$, and $C_{\operatorname{maxu}}$ on Days 1 and 22 were natural log-transformed and analyzed using a one-way analysis of variance (ANOVA) model with renal function group as a fixed effect to compare each renal impairment group (mild, moderate, severe) with the normal renal function group determined using measured BSA-normalized eGFR values at enrollment. Relationships between renal function (measured by within-patient mean values of BSA-normalized eGFR, absolute eGFR, and $\mathrm{CL}_{\mathrm{cr}}$ ) and Day $22 \mathrm{PK}$ parameters $\left(\mathrm{AUC}_{0-24}, \mathrm{AUC}_{0-24 \mathrm{u}} \mathrm{CL} / \mathrm{F}\right.$, and $\mathrm{CL}_{\mathrm{u}} / \mathrm{F}$ ) were determined by a linear regression model. Renal function input was based on the within-patient mean of all assessments performed from the enrollment visit through the Day 22 PK collection visit.

Safety analyses were performed using the safety population, which included all patients who received any amount of talazoparib. Safety data were summarized descriptively by renal function group.

\section{Results}

\subsection{Patient Demographics}

A total of 34 patients $(9,9,8$, and 8 patients with normal renal function, mild, moderate, and severe renal impairment, respectively) received talazoparib during the study. All patients were included in the safety population. The PK analysis population comprised 28 (82.4\%) patients, following the exclusion of two patients who discontinued the study due to a grade 3/4 TEAE and four patients who did not meet PK evaluability criteria (two patients with normal renal function and two patients with mild renal impairment). All 28 patients in the PK population continued talazoparib treatment in the OLE study. The baseline demographic and clinical characteristics of the PK analysis population are presented in Table 1, and that of the safety population are provided in Electronic Supplementary Table 1.

\subsection{Pharmacokinetics}

Considering the prolonged half-life of talazoparib (101.3 h) [12] and that multiple-dose PK parameters were the primary endpoints of this study, only PK results after multiple administrations of talazoparib (Day 22) are presented in this manuscript (Table 2). The PK results after a single dose of talazoparib (Day 1) are provided in Electronic Supplementary Table 2 for completeness. Geometric mean $f_{\mathrm{u}}$ values were similar across the renal function groups (Table 2). Because worsening renal function did not have an observed impact on talazoparib $f_{\mathrm{u}}$, the results obtained from the analyses of 
talazoparib total plasma PK parameters were used to derive the conclusion of the study.

Following administration of multiple oral doses of talazoparib, the median talazoparib plasma concentrations were similar between patients with normal renal function and those with mild renal impairment (Fig. 1); however, the median concentrations of talazoparib increased in patients with moderate and severe renal impairment.

As shown in Table 2, the median $T_{\max }$ was similar across the renal function groups, except in patients with severe renal impairment, where it is approximately double that in patients with normal renal function. In general, plasma talazoparib exposure increased with worsening renal function. Geometric mean $\mathrm{AUC}_{0-24}$ and $C_{\max }$ were higher in patients with renal impairment than in those with normal renal function (Table 2; Electronic Supplementary Fig. 1). A similar trend was observed in the median $R_{\text {ac }}$ of talazoparib (Table 2).
Results of the statistical comparisons of talazoparib $\mathrm{AUC}_{0-24}$ and $C_{\text {max }}$ from the ANOVA analyses are summarized in Table 3. Based on the ratios (impaired/normal) of geometric means for $\mathrm{AUC}_{0-24}$, total talazoparib exposure increased by $12.2 \%$ (mild impairment), $43.0 \%$ (moderate impairment), and $163.3 \%$ (severe impairment) after multiple doses of talazoparib relative to patients with normal renal function. Peak talazoparib exposure based on ratios of adjusted geometric means for $C_{\max }$ increased by $11.1 \%$ (mild impairment), 31.6\% (moderate impairment), and 89.3\% (severe impairment) after multiple doses of talazoparib relative to patients with normal renal function (Table 3).

Linear regression analysis of natural log-transformed $\mathrm{AUC}_{0-24}$ and $\mathrm{CL} / \mathrm{F}$ versus $\mathrm{CL}_{\mathrm{cr}}$ is shown in Fig. 2. Using the midpoint of each renal impairment group $(75 \mathrm{~mL} / \mathrm{min}$ for mild impairment, $45 \mathrm{~mL} / \mathrm{min}$ for moderate impairment, and $22.5 \mathrm{~mL} / \mathrm{min}$ for severe impairment) as an input into the linear regression model $\left(\mathrm{Ln}\left[\mathrm{AUC}_{0-24}\right]=5.69-0.01 \bullet \mathrm{CL}_{\mathrm{cr}}[\mathrm{mL} /\right.$ $\mathrm{min}]$ ), the predicted $\mathrm{AUC}_{0-24}$ values for each group on Day

Table 1 Patient demographics and baseline clinical characteristics (PK analysis population)

\begin{tabular}{|c|c|c|c|c|c|}
\hline & $\begin{array}{l}\text { Normal renal func- } \\
\text { tion }[n=6]\end{array}$ & $\begin{array}{l}\text { Mild renal impair- } \\
\text { ment }[n=7]\end{array}$ & $\begin{array}{l}\text { Moderate renal } \\
\text { impairment }[n=8]\end{array}$ & $\begin{array}{l}\text { Severe renal impair- } \\
\text { ment }[n=7]\end{array}$ & All patients $[n=28]$ \\
\hline Age, years [median (range)] & $59.0(52-70)$ & $66.0(55-78)$ & $64.5(56-74)$ & $77.0(59-88)$ & $66.5(52-88)$ \\
\hline \multicolumn{6}{|l|}{ Sex, $n(\%)$} \\
\hline Female & $3(50.0)$ & $5(71.4)$ & $6(75.0)$ & $4(57.1)$ & $18(64.3)$ \\
\hline Male & $3(50.0)$ & $2(28.6)$ & $2(25.0)$ & $3(42.9)$ & $10(35.7)$ \\
\hline \multicolumn{6}{|l|}{ Race, $n(\%)$} \\
\hline White & $5(83.3)$ & $7(100.0)$ & $7(87.5)$ & $6(85.7)$ & $25(89.3)$ \\
\hline Black or African American & 0 & 0 & 0 & 0 & 0 \\
\hline Asian & $1(16.7)$ & 0 & 0 & $1(14.3)$ & $2(7.1)$ \\
\hline Other & 0 & 0 & $1(12.5)$ & 0 & $1(3.6)$ \\
\hline \multicolumn{6}{|l|}{ Ethnicity, $n(\%)$} \\
\hline Hispanic or Latino & 0 & 0 & $1(12.5)$ & 0 & $1(3.6)$ \\
\hline Not-Hispanic or Latino & $6(100.0)$ & $7(100.0)$ & $7(87.5)$ & $7(100.0)$ & $27(96.4)$ \\
\hline Weight, kg [median (range)] & $71.9(43.8-96.4)$ & $63.4(50.1-91.4)$ & $84.8(55.1-115.0)$ & $70.4(51.2-82.3)$ & $73.4(43.8-115.0)$ \\
\hline Height, cm [median (range)] & $162(150-188)$ & $170(154-176)$ & $164(152-175)$ & $163(146-183)$ & $164(146-188)$ \\
\hline BMI, $\mathrm{kg} / \mathrm{m}^{2}$ [median (range)] & $25.8(19.5-33.8)$ & $22.1(17.3-35.4)$ & $30.8(20.1-42.3)$ & $25.9(21.9-33.4)$ & $25.9(17.3-42.3)$ \\
\hline \multicolumn{6}{|l|}{ Primary cancer site, $n(\%)$} \\
\hline Breast & $1(16.7)$ & 0 & 0 & 0 & $1(3.6)$ \\
\hline Ovary & $2(33.3)$ & $2(28.6)$ & $4(50.0)$ & $3(42.9)$ & $11(39.3)$ \\
\hline Prostate & $2(33.3)$ & 0 & $2(25.0)$ & $1(14.3)$ & $5(17.9)$ \\
\hline Other $^{\mathrm{a}}$ & $1(16.7)$ & $5(71.4)$ & $2(25.0)$ & $3(42.9)$ & $11(39.3)$ \\
\hline \multicolumn{6}{|l|}{ ECOG performance, $n(\%)$} \\
\hline 0 & $2(33.3)$ & $2(28.6)$ & $1(12.5)$ & $1(14.3)$ & $6(21.4)$ \\
\hline 1 & $3(50.0)$ & $5(71.4)$ & $5(62.5)$ & $4(57.1)$ & $17(60.7)$ \\
\hline 2 & $1(16.7)$ & 0 & $2(25.0)$ & $2(28.6)$ & $5(17.9)$ \\
\hline
\end{tabular}

Data are expressed as $n(\%)$ unless otherwise specified

$B M I$ body mass index, ECOG Eastern Cooperative Oncology Group, $P K$ pharmacokinetic

${ }^{a}$ Other primary cancer sites include the endometrium (three patients [10.7\%]), gastric, biliary, pleura, sarcoma, bladder, colon, urinary bladder, and submandibular (one patient each [3.6\%]) 
Table 2 Descriptive summary of plasma and urine talazoparib PK parameters following administration of multiple oral doses of talazoparib, by renal function group (Day 22, PK analysis population)

\begin{tabular}{|c|c|c|c|c|}
\hline & \multicolumn{4}{|c|}{ Parameter summary statistics ${ }^{\mathrm{a}}$ by renal function group } \\
\hline & Normal renal function & Mild renal impairment & Moderate renal impairment & Severe renal impairment \\
\hline Plasma PK & $n=6$ & $n=7$ & $n=8^{\mathrm{b}}$ & $n=7$ \\
\hline $\mathrm{AUC}_{0-24}[\mathrm{ng} \bullet \mathrm{h} / \mathrm{mL}]$ & $94.88(27)$ & $106.5(40)$ & $135.7(45)$ & $249.8(30)$ \\
\hline$C_{\max }[\mathrm{ng} / \mathrm{mL}]$ & $8.609(35)$ & $9.568(58)$ & $11.33(45)$ & $16.30(34)$ \\
\hline$C_{\text {trough }}[\mathrm{ng} / \mathrm{mL}]$ & $2.172(34)$ & $2.680(52)$ & $3.893(45)$ & $7.917(44)$ \\
\hline$T_{\max }[\mathrm{h}]$ & $1.50(0.97-4.00)$ & $2.00(0.50-4.00)$ & $1.49(0.47-4.02)$ & $3.88(0.50-8.00)$ \\
\hline $\mathrm{CL} / \mathrm{F}[\mathrm{L} / \mathrm{h}]$ & $5.270(27)$ & $4.698(40)$ & $3.687(45)$ & $2.000(30)$ \\
\hline$R_{\mathrm{ac}}$ & $4.465(3.44-15.6)$ & $4.070(3.05-9.15)$ & $5.970(3.97-10.6)$ & $7.170(5.66-14.9)$ \\
\hline$f_{\mathrm{u}}[\%]$ & $29.85(24)$ & $31.54(10)$ & $29.09(9)$ & $32.49(30)$ \\
\hline $\mathrm{AUC}_{0-24 \mathrm{u}}[\mathrm{ng} \bullet \mathrm{h} / \mathrm{mL}]$ & $28.33(32)$ & $33.59(31)$ & $39.47(47)$ & $81.17(36)$ \\
\hline$C_{\operatorname{maxu}}[\mathrm{ng} / \mathrm{mL}]$ & $2.570(42)$ & $3.019(47)$ & $3.295(48)$ & $5.296(30)$ \\
\hline $\mathrm{CL}_{\mathrm{u}} / F[\mathrm{~L} / \mathrm{h}]$ & $17.66(32)$ & $14.89(31)$ & $12.68(47)$ & $6.164(36)$ \\
\hline Urine PK & $n=5$ & $n=7$ & $n=7$ & $n=7^{\mathrm{c}}$ \\
\hline $\mathrm{Ae}_{0-24}[\mathrm{mg}]$ & $0.258(23)$ & $0.232(20)$ & $0.164(54)$ & $0.201(34)$ \\
\hline $\mathrm{Ae}_{20-4}[\%]$ & $51.7(23)$ & $46.4(20)$ & $32.8(54)$ & $40.1(34)$ \\
\hline $\mathrm{CL}_{\mathrm{r}}[\mathrm{L} / \mathrm{h}]$ & $2.74(37)$ & $2.18(57)$ & $1.33(97)$ & $0.709(35)$ \\
\hline
\end{tabular}

$A e_{0-24}$ amount of drug excreted in urine from time 0 to $24 \mathrm{~h}$ postdose, $A U C_{0-24}$ area under the concentration-time curve from 0 to $24 \mathrm{~h}, A U C_{0-24 u}$ unbound $\mathrm{AUC}_{0-24}, C L / F$ apparent oral clearance, $C L_{l} / F$ unbound $\mathrm{CL} / \mathrm{F}, C L_{r}$ renal clearance, $C_{\text {max }}$ maximum observed plasma concentration, $C_{\text {maxu }}$ unbound $\mathrm{C}_{\max }, C_{\text {trough }}$ plasma trough (predose) concentration, $C V \%$ percentage coefficient of variation, $f_{u}$ fraction of unbound drug in plasma, $P K$ pharmacokinetics, $R_{a c}$ accumulation ratio, $T_{\max }$ time to $\mathrm{C}_{\max }$

${ }^{\mathrm{a}}$ Geometric mean (geometric $\mathrm{CV} \%$ ) for all parameters except median (range) for $T_{\max }$ and $\mathrm{R}_{\mathrm{ac}}$

${ }^{\mathrm{b}} n=7$ for $\mathrm{R}_{\mathrm{ac}}$

${ }^{\mathrm{c}} n=5$ for $\mathrm{CL}_{\mathrm{r}}$

Fig. 1 Median talazoparib plasma concentration following multiple oral doses of talazoparib in patients with varying degrees of renal function (Day 22, PK population). $P K$ pharmacokinetics

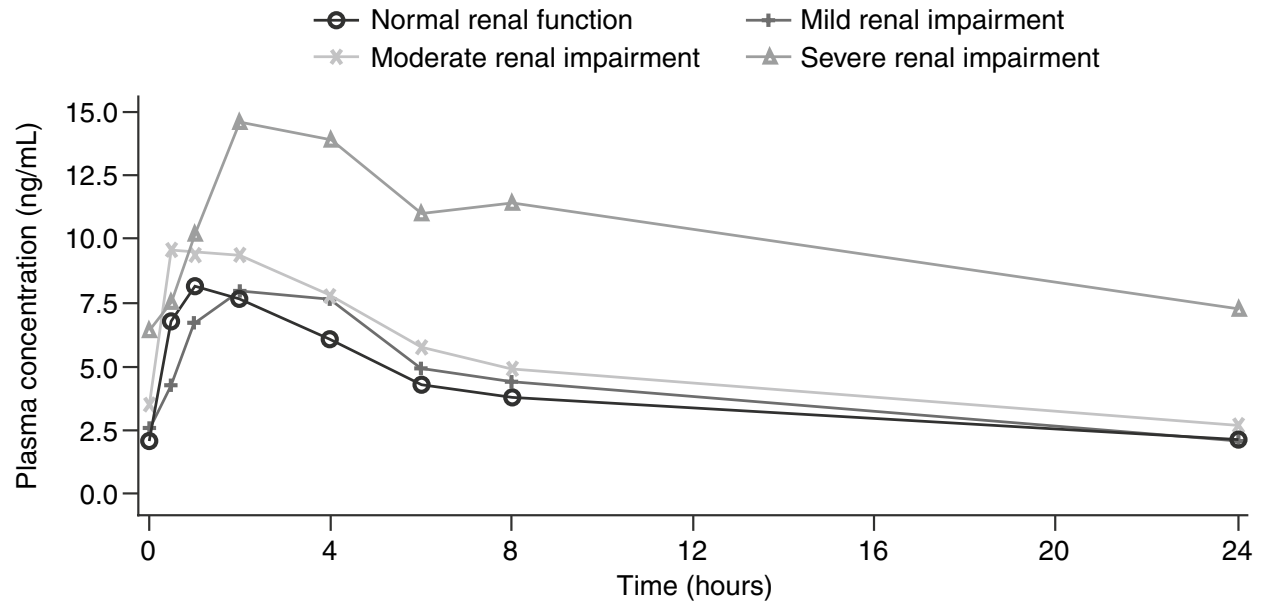

22 would be $139.8 \mathrm{ng} \bullet \mathrm{h} / \mathrm{mL}, 188.7 \mathrm{ng} \bullet \mathrm{h} / \mathrm{mL}$, and 236.3 $\mathrm{ng} \bullet \mathrm{h} / \mathrm{mL}$, respectively. These predicted $\mathrm{AUC}_{0-24}$ values correspond to increases in talazoparib $\mathrm{AUC}_{0-24}$ of approximately $16.2 \%$ (mild impairment), 56.8\% (moderate impairment), and $96.4 \%$ (severe impairment) versus normal renal function. Similar results were obtained when other assessments of renal function were used (BSA-normalized eGFR and absolute eGFR; Electronic Supplementary Table 3).
Urinary excretion of talazoparib accounted for $51.7 \%$ of the administered dose in the normal renal function group and $32.8-46.4 \%$ of the dose in patients with renal impairment (Table 2). The $\mathrm{CL}_{\mathrm{r}}$ of talazoparib decreased with worsening renal impairment (Table 2). 
Table 3 Statistical summary of renal function group comparisons of talazoparib total plasma PK parameters following administration of multiple oral doses of talazoparib (Day 22, PK analysis population)

\begin{tabular}{llccr}
\hline Parameter & Comparison (Test/Reference) & \multicolumn{2}{l}{ Geometric means } & \multirow{2}{*}{$\begin{array}{l}\text { Ratio of geometric } \\
\text { means, \% (90\% CI) }\end{array}$} \\
\cline { 3 - 3 } & & Test & Reference & \\
\hline $\mathrm{AUC}_{0-24}[\mathrm{ng} \bullet \mathrm{h} / \mathrm{mL}]$ & Mild/normal & 106.5 & 94.88 & $112.20(80.06-157.26)$ \\
& Moderate/normal & 135.7 & 94.88 & $142.98(103.03-198.42)$ \\
& Severe/normal & 249.8 & 94.88 & $263.32(187.88-369.06)$ \\
$C_{\max }[\mathrm{ng} / \mathrm{mL}]$ & Mild/normal & 9.568 & 8.609 & $111.13(74.40-166.01)$ \\
& Moderate/normal & 11.33 & 8.609 & $131.57(89.12-194.25)$ \\
& Severe/normal & 16.30 & 8.609 & $189.32(126.74-282.80)$ \\
\hline
\end{tabular}

$A U C_{0-24}$ area under the concentration-time curve from 0 to $24 \mathrm{~h}, C I$ confidence interval, $C_{\max }$ maximum observed plasma concentration, $P K$ pharmacokinetics
Fig. 2 Regression and $90 \%$ CI of natural log-transformed plasma talazoparib a $\mathrm{AUC}_{0-24}$ and $\mathbf{b} \mathrm{CL} / \mathrm{F}$ versus $\mathrm{CL}_{\mathrm{cr}}(\mathrm{mL} /$ min) following administration of multiple oral doses of talazoparib in patients with varying degrees of renal impairment (Day 22, PK analysis population). $A U C_{0-24}$ area under the concentration-time curve from 0 to $24 \mathrm{~h}, C I$ confidence interval, $C L / F$ apparent oral clearance, $C L_{c r}$ creatinine clearance, $P K$ pharmacokinetics

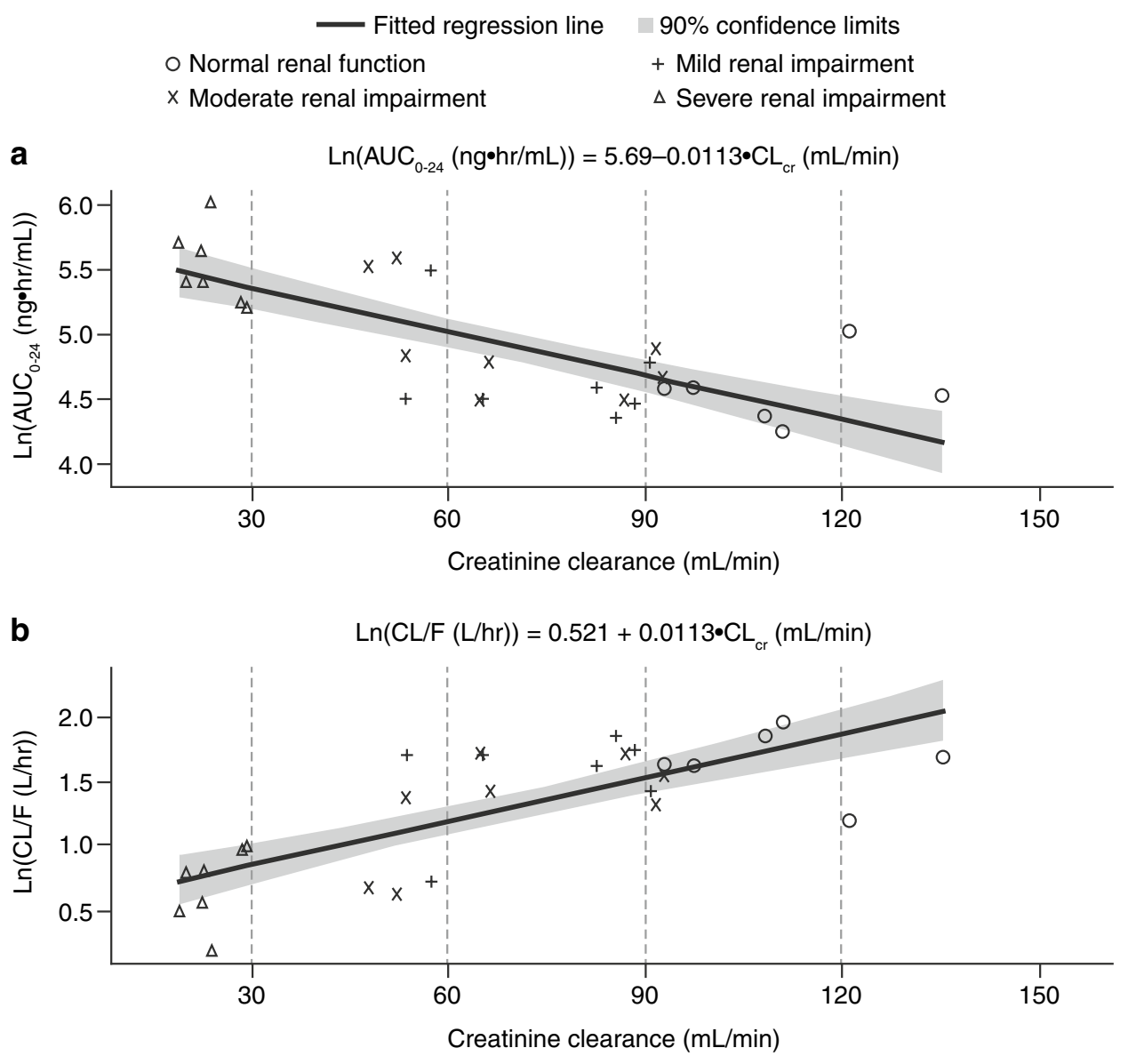

\subsection{Safety}

Overall, 30 patients $(88.2 \%)$ reported at least one TEAE (Table 4). There were no unexpected safety findings in this study and reported TEAEs were considered consistent with the disease under study and the established safety profile associated with talazoparib treatment. Overall, there were no notable differences in the TEAE profile between patients with normal renal function and patients with mild, moderate, or severe renal impairment. The frequency of all-causality non-serious TEAEs of any grade were similar across all renal function groups; however, the frequency of all-causality SAEs was higher in the severe renal impairment group, although none of the SAEs reported in the study were considered related to talazoparib treatment by the investigator.

The most commonly reported treatment-related TEAEs were fatigue $(n=6,17.6 \%)$, anemia $(n=5,14.7 \%)$, and nausea $(n=4,11.8 \%)$. Grade 3 or higher TEAEs occurred in five patients (14.7\%). Grade 3/4 TEAEs considered related to talazoparib were anemia and thrombocytopenia $(n=2$, $5.9 \%$ each), and fatigue $(n=1,2.9 \%)$. Five treatment-emergent SAEs were reported in three patients $(8.8 \%)$, although 
Table 4 Summary of TEAEs by renal function group (safety population)

\begin{tabular}{|c|c|c|c|c|c|}
\hline$n(\%)$ & $\begin{array}{l}\text { Normal renal } \\
\text { function }[n=9]\end{array}$ & $\begin{array}{l}\text { Mild renal } \\
\text { impairment } \\
{[n=9]}\end{array}$ & $\begin{array}{l}\text { Moderate renal } \\
\text { impairment }[n=8]\end{array}$ & $\begin{array}{l}\text { Severe renal } \\
\text { impairment } \\
{[n=8]}\end{array}$ & All patients $[n=34]$ \\
\hline Patients with any TEAE & $7(77.8)$ & $8(88.9)$ & $8(100)$ & $7(87.5)$ & $30(88.2)$ \\
\hline $\begin{array}{l}\text { Patients with any TEAE of Grade } 3 \text { or } \\
\text { higher }\end{array}$ & $2(22.2)$ & 0 & $1(12.5)$ & $2(25.0)$ & $5(14.7)$ \\
\hline Patients with any serious TEAE & $1(11.1)$ & 0 & 0 & $2(25.0)$ & $3(8.8)$ \\
\hline Patients with any TEAE leading to death & $1(11.1)$ & 0 & 0 & 0 & $1(2.9)$ \\
\hline $\begin{array}{l}\text { Patients with temporary and permanent } \\
\text { study drug discontinuation due to TEAE }\end{array}$ & $2(22.2)$ & $1(11.1)$ & 0 & $2(25.0)$ & $5(14.7)$ \\
\hline \multicolumn{6}{|c|}{ Treatment-related TEAEs that occurred in $\geq 5 \%$ of patients, by preferred term } \\
\hline Fatigue & $1(11.1)$ & $3(33.3)$ & $1(12.5)$ & $1(12.5)$ & $6(17.6)$ \\
\hline Anemia & $2(22.2)$ & $1(11.1)$ & $1(12.5)$ & $1(12.5)$ & $5(14.7)$ \\
\hline Nausea & $2(22.2)$ & - & $2(25.0)$ & - & $4(11.8)$ \\
\hline Diarrhea & $1(11.1)$ & - & $1(12.5)$ & $1(12.5)$ & $3(8.8)$ \\
\hline Thrombocytopenia & $1(11.1)$ & - & $1(12.5)$ & $1(12.5)$ & $3(8.8)$ \\
\hline AST increased & - & - & - & $2(25.0)$ & $2(5.9)$ \\
\hline Decreased appetite & - & - & - & $2(25.0)$ & $2(5.9)$ \\
\hline Dizziness & - & $2(22.2)$ & - & - & $2(5.9)$ \\
\hline Vomiting & $1(11.1)$ & - & - & $1(12.5)$ & $2(5.9)$ \\
\hline
\end{tabular}

$A S T$ aspartate aminotransferase, TEAE treatment-emergent adverse event

none were considered to be related to talazoparib. One patient (2.9\%) died on Day 27 due to 'condition aggravated'; the patient had normal renal function and the event was not considered to be related to talazoparib by the investigator.

Two patients $(5.9 \%)$ permanently discontinued talazoparib treatment due to grade 3/4 TEAEs. One patient with severe renal impairment discontinued treatment after receiving the last dose on Day 21 due to grade 3 dehydration, which was not considered related to treatment. The second patient had normal renal function and castration-resistant metastatic prostate cancer with extensive bone metastases, and was heavily pretreated, including palliative radiotherapy prior to study enrollment. The patient discontinued treatment after receiving the last dose on Day 13 due to grade 4 thrombocytopenia considered to be related to treatment, which resolved to grade 1 after 14 days. A further three patients $(8.8 \%)$ had a dose interruption due to TEAEs, which included grade 3 anemia (in a patient with normal renal function), grade 2 fatigue (in a patient with mild renal impairment), and grade 3 dyspnea (in a patient with severe renal impairment); the patient with mild renal impairment also required a dose reduction to $0.25 \mathrm{mg} /$ day on Day 22 due to grade 2 fatigue.

\section{Discussion}

Consistent with the major role of renal excretion in the clearance of talazoparib [10,13], results from this phase I study indicate that talazoparib exposure increased, and clearance of the drug decreased, with worsening renal function. The linear regression analyses, used to assess the relationship between renal function and the $\mathrm{AUC}_{0-24}$ and $\mathrm{CL} / \mathrm{F}$ of talazoparib, generated consistent results, regardless of whether renal function was assessed by BSA-normalized eGFR, absolute eGFR, or $\mathrm{CL}_{\mathrm{cr}}$, and thereby support the interchangeability of the renal function assessments when providing dosing recommendations. These results are consistent with a previous analysis showing that drug dosing regimens derived using either MDRD or Cockcroft-Gault for classification of renal function were concordant in $88 \%$ of patients [16]. All three regression models provided the same numeric conclusion with regard to the expected ratios of exposure across the renal function groups, although the linear regression that used $\mathrm{CL}_{\mathrm{cr}}$ as the renal function input provided the best fit to the data, as measured by the adjusted coefficient of determination.

The projected increase in talazoparib exposure relative to patients with normal renal function using linear regression was similar to that of ANOVA analyses for patients with mild (16.2\% vs. $12.2 \%$, respectively) or moderate (56.8\% vs. $43.0 \%$, respectively) renal impairment, but was numerically lower than with ANOVA for patients with severe renal impairment (96.4\% vs. $163.3 \%$, respectively). Nevertheless, 
the results of ANOVA analyses (categorical analyses across predefined renal function groups) and linear regression models (treating renal function as a continuous covariate) were generally consistent with those of the population PK analysis for patients with mild and moderate renal impairment [13].

Current dosing recommendations for patients with mild and moderate renal impairment were based on the population PK analysis of data pooled across prior talazoparib studies conducted in patients with advanced cancers, which evaluated the effect of renal impairment on the PK of talazoparib $[4,5,14]$. Based on the results from that analysis, which indicated that talazoparib CL/F was decreased by approximately $15 \%$ and $38 \%$ (corresponding to increases in steady-state $\mathrm{AUC}_{0-24}$ of approximately $18 \%$ and $61 \%$ ) in patients with mild and moderate renal impairment, respectively, talazoparib dose was reduced to $0.75 \mathrm{mg}$ for patients with moderate renal impairment, while no dose adjustment was required for patients with mild renal impairment. However, due to insufficient data, no dosing recommendations were provided for patients with severe renal impairment. The above dosing recommendations use $\mathrm{CL}_{\mathrm{cr}}$ to define renal impairment categories rather than eGFR as used in this study. The linear regression analyses from this study support the interchangeability of these renal function assessments (BSA-normalized eGFR, absolute eGFR, and $\mathrm{CL}_{\mathrm{cr}}$ ). Based on the predicted $\mathrm{AUC}_{0-24}$ values using the linear regression model where talazoparib exposure increased by $56.8 \%$ and $96.4 \%$ in patients with moderate and severe renal impairment, respectively, dose reduction to $0.75 \mathrm{mg}$ in patients with moderate renal impairment and to $0.5 \mathrm{mg}$ in patients with severe renal impairment will provide exposure comparable to $1 \mathrm{mg}$ in patients with normal renal function.

Talazoparib administered at a once-daily oral dose of $0.5 \mathrm{mg}$ for 22 days was generally tolerated in patients with advanced solid tumors, with no unexpected safety findings reported. TEAEs were consistent with the disease characteristics and the known safety profile of talazoparib [8], and there were no notable differences in TEAEs across all renal function groups. Although the frequency of all-cause SAEs was higher in the severe renal impairment group than in other groups, none of the SAEs were considered to be related to talazoparib treatment by the investigators.

One of the study limitations includes the use of a lower dose of talazoparib $(0.5 \mathrm{mg})$ when compared to the standard therapeutic and maximum tolerated dose $(1 \mathrm{mg})$; however, the lower dose was chosen to account for the fact that talazoparib exposure was likely to be higher in patients with renal impairment versus those with normal renal function. As talazoparib exposure has been shown to increase in a doseproportional manner across the dose range of $0.025-1.1 \mathrm{mg}$ at steady state, the results obtained in this study can be generalized to other talazoparib doses. In addition, missed doses may impact steady-state talazoparib exposure, and the impact of missed doses could theoretically have been greater in patients with more severe renal impairment. However, only four patients failed to meet the PK evaluability criteria and were excluded from PK analyses; all had either normal renal function or mild renal impairment. There was also no obvious pattern in dose interruptions by the renal function group.

\section{Conclusions}

In this study, plasma talazoparib total exposure was increased by $12.2 \%, 43.0 \%$, and $163.3 \%$ in patients with mild, moderate, and severe renal impairment, respectively, relative to those with normal renal function. The apparent renal clearance of talazoparib decreased with worsening renal function. Talazoparib was generally tolerated, with no notable differences in the TEAE profile between patients with normal renal function and those with mild, moderate, or severe renal impairment. Overall, this study confirms current dosing recommendations in patients with mild and moderate renal impairment $(1 \mathrm{mg}$ and $0.75 \mathrm{mg}$ once daily, respectively), and suggests that a lower starting dose of $0.5 \mathrm{mg}$ once daily (the dose used in this study) should be considered for patients with severe renal impairment.

Supplementary Information The online version contains supplementary material available at https://doi.org/10.1007/s40262-020-00983-y.

Acknowledgments Medical writing support was provided by Katharine Howe, PhD, of CMC AFFINITY, McCann Health Medical Communications, and was funded by Pfizer.

\section{Declarations}

Funding This study was sponsored by Medivation, which was acquired by Pfizer in September 2016.

Conflicts of Interest Chandrasekar Durairaj, Jayeta Chakrabarti, Anna Plotka, Justin Hoffman, Haihong Shi, and Diane D. Wang are employees of Pfizer and receive stock and stock options as part of their employment. Cristiano Ferrario has received consulting fees from Astellas, Bayer, Eli Lilly, Novartis, Pfizer, and Roche, and research funding from Astellas, AstraZeneca, Merck, Novartis, Pfizer, Roche/ Genentech, Sanofi, Seattle Genetics, and Zymeworks. Holger W. Hirte has no disclosures to declare. Sunil Babu has received honoraria from Alexion Pharmaceuticals, AstraZeneca, Bayer, Bristol-Myers Squibb, and Lilly; consulting fees from Alexion Pharmaceuticals, Argenx, AstraZeneca, Boehringer Ingelheim, and Bristol-Myers Squibb; speaker fees from Alexion Pharmaceuticals; research/grant funding (through his institution) from Abbvie, Alexion Pharmaceuticals, Amgen, Argenx, AstraZeneca/Medimmune, Bristol-Myers Squibb, Genentech/ Roche, Janssen Oncology, Lilly, Merck, Nektar, Novartis, Sanofi, Syndax, and TG Therapeutics; and travel/accommodation expenses from Alexion Pharmaceuticals, Bristol-Myers Squibb, and Lilly. Sarina A. Piha-Paul has received research/grant funding (through her institution) from AbbVie, Inc., Alkermes, Aminex Therapeutics, Amphivena Therapeutics, Inc., BioMarin Pharmaceutical, Inc., Boehringer Ingel- 
heim, Bristol Myers Squib, Cerulean Pharma Inc., Chugai Pharmaceutical Co., Ltd, Curis, Inc., Daichi Sanko, Eli Lilly, Five Prime Therapeutics, Genmab A/S, GlaxoSmithKline, Helix BioPharma Corp., Incyte Corp., Jacobio Pharmaceuticals Co., Ltd, Medimmune, LLC., Medivation, Inc., Merck Sharp and Dohme Corp., NewLink Genetics Corporation/Blue Link Pharmaceuticals, Novartis Pharmaceuticals, Pieris Pharmaceuticals, Inc., Pfizer, Principia Biopharma, Inc., Puma Biotechnology, Inc., Rapt Therapeutics, Inc., Seattle Genetics, Taiho Oncology, Tesaro, Inc., TransThera Bio, and XuanZhu Biopharma. NCI/NIH Core Grant, P30CA016672 (CCSG Shared Resources).

Ethics Approval The study protocol was conducted in accordance with the International Conference on Harmonisation Guidelines for Good Clinical Practice and the Declaration of Helsinki. The protocol also complied with the laws and regulations, as well as any applicable guidelines, of the countries where the study was conducted. The protocol was approved by the Independent Ethics Committee or Institutional Review Board at each of the investigational centers participating in the study.

Consent to Participate All patients provided written informed consent prior to undergoing any study-specific procedure.

Consent for publication Not applicable.

Availability of Data and Material Upon request, and subject to certain criteria, conditions, and exceptions (see https://www.pfizer.com/scien ce/clinical-trials/trial-data-and-results for more information), Pfizer will provide access to individual de-identified participant data from Pfizer-sponsored global interventional clinical studies conducted for medicines, vaccines, and medical devices (1) for indications that have been approved in the US and/or EU, or (2) in programs that have been terminated (i.e. development for all indications has been discontinued). Pfizer will also consider requests for the protocol, data dictionary, and statistical analysis plan. Data may be requested from Pfizer trials 24 months after study completion. The de-identified participant data will be made available to researchers whose proposals meet the research criteria and other conditions, and for which an exception does not apply, via a secure portal. To gain access, data requestors must enter into a data access agreement with Pfizer.

Code availability Not applicable.

Author Contributions All authors contributed to the design of the study and/or assisted with the data analysis/interpretation of the data. All authors assisted in the preparation of the manuscript, reviewed the manuscript, and provided their approval for submission. All authors agree to be accountable for all aspects of the work presented.

Open Access This article is licensed under a Creative Commons Attribution-NonCommercial 4.0 International License, which permits any non-commercial use, sharing, adaptation, distribution and reproduction in any medium or format, as long as you give appropriate credit to the original author(s) and the source, provide a link to the Creative Commons licence, and indicate if changes were made. The images or other third party material in this article are included in the article's Creative Commons licence, unless indicated otherwise in a credit line to the material. If material is not included in the article's Creative Commons licence and your intended use is not permitted by statutory regulation or exceeds the permitted use, you will need to obtain permission directly from the copyright holder. To view a copy of this licence, visit http://creativecommons.org/licenses/by-nc/4.0/.

\section{References}

1. Shen Y, Rehman FL, Feng Y, Boshuizen J, Bajrami I, Elliott R, et al. BMN 673, a novel and highly potent PARP1/2 inhibitor for the treatment of human cancers with DNA repair deficiency. Clin Cancer Res. 2013;19(18):5003-15.

2. Lord CJ, Ashworth A. PARP inhibitors: Synthetic lethality in the clinic. Science. 2017;355(6330):1152-8.

3. Murai J, Huang SY, Renaud A, Zhang Y, Ji J, Takeda S, et al. Stereospecific PARP trapping by BMN 673 and comparison with olaparib and rucaparib. Mol Cancer Ther. 2014;13(2):433-43.

4. European Medicines Agency. TALZENNA® (talazoparib) Summary of Product Characteristics. July 2019. https://www.ema. europa.eu/en/medicines/human/EPAR/talzenna. Accessed 26 Jun 2020.

5. Pfizer Inc. TALZENNA® (talazoparib) [prescribing information]. March 2020. http://labeling.pfizer.com/ShowLabeli ng.aspx?id=11046. Accessed 26 Jun 2020.

6. Ettl J, Quek RGW, Lee K-H, Rugo HS, Hurvitz S, Gonçalves A, et al. Quality of life with talazoparib versus physician's choice of chemotherapy in patients with advanced breast cancer and germline BRCA1/2 mutation: patient-reported outcomes from the EMBRACA phase III trial. Ann Oncol. 2018;29(9):1939-47.

7. Litton JK, Rugo HS, Ettl J, Hurvitz SA, Gonçalves A, Lee K-H, et al. Talazoparib in patients with advanced breast cancer and a germline BRCA mutation. N Engl J Med. 2018;379(8):753-63.

8. Hurvitz SA, Gonçalves A, Rugo HS, Lee K-H, Fehrenbacher L, Mina LA, et al. Talazoparib in patients with a germline $B R C A$ -mutated advanced breast cancer: detailed safety analyses from the phase III EMBRACA Trial. Oncologist. 2020;25:e439-50.

9. Hoffman J, Chakrabarti J, Plotka A, Milillo Naraine A, Kanamori $\mathrm{D}$, Moroose R, et al. Talazoparib has no clinically relevant effect on QTc interval in patients with advanced solid tumors. Anticancer Drugs. 2019;30(5):523-32.

10. Yu Y, Chung CH, Plotka A, Quinn K, Shi H, Papai Z, et al. A Phase 1 mass balance study of (14) C-labeled talazoparib in patients with advanced solid tumors. J Clin Pharmacol. 2019;59(9):1195-203.

11. de Bono J, Ramanathan RK, Mina L, Chugh R, Glaspy J, Rafii S, et al. Phase I, dose-escalation, two-part trial of the PARP inhibitor talazoparib in patients with advanced germline $B R C A 1 / 2$ mutations and selected sporadic cancers. Cancer Discov. 2017;7(6):620-9.

12. Elmeliegy M, Láng I, Smolyarchuk EA, Chung C-H, Plotka A, Shi $\mathrm{H}$, et al. Evaluation of the effect of P-glycoprotein inhibition and induction on talazoparib disposition in patients with advanced solid tumours. Br J Clin Pharmacol. 2020;86(4):771-8.

13. Yu Y, Durairaj C, Shi H, Wang DD. Population pharmacokinetics of talazoparib in patients with advanced cancer. J Clin Pharmacol. 2020;60(2):218-28.

14. European Medicines Agency. Assessment report: Talzenna [procedure no. EMEA/H/C/004674/0000]. 2019 [cited 26 Jun 2020]. Available at: https://www.ema.europa.eu/en/documents/asses sment-report/talzenna-epar-public-assessment-report_en.pdf

15. European Medicines Agency. Guideline on the evaluation of the pharmacokinetics of medicinal products in patients with decreased renal function. 2015. https://www.ema.europa.eu/en/documents/ scientific-guideline/guideline-evaluation-pharmacokinetics-medic inal-products-patients-decreased-renal-function_en.pdf. Accessed 26 June 2020.

16. Park EJ, Wu K, Mi Z, Dong T, Lawrence JP, Ko CW, et al. A systematic comparison of Cockcroft-Gault and Modification of Diet in Renal Disease equations for classification of kidney dysfunction and dosage adjustment. Ann Pharmacother. 2012;46(9):1174-87. 